\begin{tabular}{|c|c|}
\hline & $\begin{array}{c}\text { International Journal of Current Research in } \\
\text { Biosciences and Plant Biology } \\
\text { BXCELLENT } \\
\text { PUBLISHERS }\end{array}$ \\
Volume $4 \bullet$ Number 4 (April-2017) $\bullet$ ISSN: 2349-8080 (Online) \\
Journal homepage: www.ijcrbp.com
\end{tabular}

\title{
Studies on Variability, Heritability and Genetic Advance Analysis for Yield and Yield Attributes of Garlic (Allium sativum L.)
}

\author{
Kuldeep Kumar ${ }^{2}$, C.N. Ram ${ }^{1}$, G. C. Yadav $^{1}$, Deepak Kumar Gautam ${ }^{2} *$ Pushpendra Kumar ${ }^{2}$ and \\ Rajesh Kumar ${ }^{3}$
}

\begin{abstract}
${ }^{1}$ Assistant Professor and ${ }^{2}$ Student, Department of Vegetable Science, Narendra Deva University of Agriculture \& Technology, Kumarganj, Faizabad-224 229 (U.P.), India

${ }^{3}$ Research Scholar, Department of Horticulture, Narendra Deva University of Agriculture \& Technology, Kumarganj, Faizabad224229 (U.P.), India
\end{abstract}

\section{*Corresponding author.}

\section{Abstract}

The experiment was conducted in Augmented Block Design at Main Experimental Station of Department of Vegetable Science, Narendra Deva University of Agriculture, Narendra Nagar (Kumarganj), Faizabad (U.P.) during Rabi 2014-15 with the following objectives (1) To find out phenotypic and genotypic coefficient of variation (PCV \& GCV), (2) to estimate heritability and genetic advance, Experimental material for the present study consisted of thirty genotypes. In single plot sized $2.0 \mathrm{X} 0.30 \mathrm{~cm}$ with the distance of 30 $\mathrm{cm}$ row to row and $10 \mathrm{~cm}$ plant to plant. Observation were recorded on plant height $(\mathrm{cm})$, leaves per plant, length of leaf $(\mathrm{cm})$, width of leaf $(\mathrm{cm})$, neck thickness of bulb $(\mathrm{cm})$, cloves per bulb, weight of clove $(\mathrm{g})$, length of clove $(\mathrm{cm})$, length of bulb $(\mathrm{cm})$, width of clove $(\mathrm{cm})$, diameter of bulb $(\mathrm{cm})$, total soluble solids $(\%)$ and bulb yield per plant (g).Analysis of variance for design of experiment indicated highly significant differences among treatments for all the characters, based on mean performance of genotypes, the NDG-43, NDG-49, Punjab Garlic, NDG-48 and NDG-45, were identified as most promising genotypes for bulb yield per plant. The higher magnitude of coefficient of variation at genotypic as well as phenotypic levels observed for leaves per plant, length of leaf, length of clove. High heritability coupled with high genetic advance in per cent of mean was recorded for number of cloves per bulb, Bulb yield per plant, weight of clove and width of leaf. It is suggested that selection for these traits will directly increase bulb yield per plant.

\section{Introduction}

Garlic (Allium sativum L.) having diploid chromosome number $2 \mathrm{n}=2 \mathrm{x}=16$ belongs to the family Amaryllidaceae (Alliaceae); known as Lahsun in Hindi, is one of the

\section{Article Info}

Accepted: 01 April 2017

Available Online: 06 April 2017

\section{Keywords}

Allium sativum L.

Garlic

Genetic advance

Genotypic coefficient of variation

Heritability

Phenotypic coefficient of variation 
medicinal herb with underground compound bulbs covered by outer white thin scales with simple smooth round stem surrounded by the bottom by tublar leaf sheath. The leaves are simple, long, flat and linear. The flowers are small and white, arranged in round umbels mixed with small bulbils. The entire umbels are enclosed in a tear-drop shaped membranous spathe. Flowers are usually sterile. The seed stalk bears terminal inflorescence, which in terms bear bulbils instead of flowers. The shoot of garlic become flat and finally aborts after the development of bulbils in the inflorescence (Kothari and Shah, 1974). A compound bulb consists of smaller bulbils or a segment called "cloves" which are formed from auxiliary bulbs of the young foliage leaves and is surrounded by a thin white or pinkish papery sheath.

The history of garlic dates back to the time immemorial original abode of garlic is said to be Central Asia and Southern Europe, especially Mediterranean region. Garlic has long been known as cultivated plant in India and China. It was carried out to the western hemisphere by the Spanish, Portuguese and French. The major garlic growing countries are Spain, Egypt, France, Mexico and Brazil. Asia shared major part in total world's garlic production. China, Korea, Spain, Egypt and USA are the major garlic growing countries. China ranks first in area and production followed by India and Korea republic.

Hence in present investigation and attempt was needed to assess the variability of important pod yield contribution traits along with the indicate of variability i.e. genotypic coefficient variation $(\mathrm{GCV})$, phenotypic coefficient variation (GCV), environment coefficient variation (ECV), Heritability IN Broad Sense $\left(h^{2} b\right)$ and genetic advance (GA) per cent of mean.

\section{Materials and methods}

The experimental material for the present investigation comprised 38 genotypes of Garlic collected from different places in India and being maintained at main experiment station in the Department of Vegetable Science, N.D. University of Agriculture and Technology, Narendra Nagar (Kumarganj), Faizabad (U.P.). Experiment was laid out in an "Augmented Block Design". The experimental field was prepared by harrowing and three cross ploughing with cultivator followed by planking. The field was well manures with FYM @ 30 tonnes per hectare 20 days before sowing. The irrigation channel was made between two blocks.
Two row of $2.0 \mathrm{~m} \times 0.30 \mathrm{~m}$ plot with the distance of $30 \mathrm{~cm}$ row to row and plant to plant $10 \mathrm{~cm}$. The cloves of each genotype were sown on 15 November in 2014. The first irrigation was done in just after sowing than irrigation was done at 15 days interval during crop growth. The recommended cultural practices and plant protection measures were applied to raise a healthy crop. All the recommended agronomic package of practices and plant protection measures were followed to raise a good crop. Observations were recorded on thirteen character viz. on plant height $(\mathrm{cm})$, leaves per plant, length of leaf $(\mathrm{cm})$, width of leaf $(\mathrm{cm})$, neck thickness of bulb $(\mathrm{cm})$, cloves per bulb, weight of clove $(\mathrm{g})$, length of clove $(\mathrm{cm})$, length of bulb $(\mathrm{cm})$, width of clove $(\mathrm{cm})$, diameter of bulb (cm), total soluble solids (\%) and bulb yield per plant $(\mathrm{g})$. The data obtained were subjected to analysis of variance to test significant according to the procedure given by Panse and Sukhatme (1967). Genetic variability calculated by the procedure given below by Burton and de Vane (1953). Heritability in broad sense $\left(\mathrm{h}^{2}\right.$ bs $)$ was calculated according to the formula suggested by Burton and de Vane (1953). Genetic advance (Ga) was estimated by the method suggested by Johnson et al. (1955).

\section{Results and discussion}

\section{Analysis of variance}

The analysis of variance for different characters is presented in (Table 1). The mean sum of square due to genotype highly significant for all the characters but length of leaf and diameter of clove are significant. The variation due to genotypes was highly significant for all characters.

\section{Mean performance of genotypes}

In order to evaluate the listed sixty genotypes including check for thirteen characters is presented in (Table 2). A wide range of variation in mean performance of genotypes was observed for all characters under study. The comparison of mean performance of sixty genotypes for thirteen traits using critical differences revealed existence of very high level of variability in the listed genotypes.

The genotype NDG-43 (34.57 g) gave highly significant higher yield as compared to others genotypes as well as check variety. The high yielding genotype NDG-43 also showed high mean performance for clove per bulb. 
Other entries with higher bulb yield per plant were NDG-49 (33.40 g), NDG-48 (32.15 g) and NDG-45 $(31.52 \mathrm{~g})$. These genotypes also showed high mean performance for some other characters besides higher bulb yield. These result suggested that above mentioned high yielding genotypes may be considered in varietal improvement programme for desired characters in garlic.

Table 1. Analysis of variance for thirteen characters in garlic.

\begin{tabular}{|c|c|c|c|c|}
\hline \multirow{3}{*}{ S. No. } & \multirow{3}{*}{ Characters } & \multicolumn{3}{|c|}{ Source of variation } \\
\hline & & Blocks & Checks & Error \\
\hline & & d.f. (7) & d.f. (3) & d.f. (21) \\
\hline 1 & Plant height $(\mathrm{cm})$ & $15.91 * *$ & $29.54 * *$ & 0.00 \\
\hline 2 & No of leaves per plant & $1.61 * *$ & $7.35^{* *}$ & 0.02 \\
\hline 3 & Length of leaf (cm) & $4.78 *$ & $20.98 * *$ & 1.13 \\
\hline 4 & Width of leaf $(\mathrm{cm})$ & $0.04 * *$ & $0.34 * *$ & 0.00 \\
\hline 5 & Neck thickness of bulb (cm) & $0.01 * *$ & $0.02 * *$ & 0.00 \\
\hline 6 & Diameter of bulb $(\mathrm{cm})$ & $0.10 * *$ & $0.94 * *$ & 0.00 \\
\hline 7 & Bulb yield per plant (g) & $28.43 * *$ & $31.61 * *$ & 0.43 \\
\hline 8 & No of cloves per bulb & $19.42 * *$ & $159.57 * *$ & 0.04 \\
\hline 9 & Weight of clove (g) & $0.05^{* *}$ & $0.32 * *$ & 0.00 \\
\hline 10 & Length of clove $(\mathrm{cm})$ & $0.17 * *$ & $0.23 * *$ & 0.01 \\
\hline 11 & Diameter of clove $(\mathrm{cm})$ & $0.17 *$ & $0.47 * *$ & 0.02 \\
\hline 12 & Length of bulb $(\mathrm{cm})$ & $0.12 * *$ & $0.31 * *$ & 0.00 \\
\hline 13 & Total soluble solids $\%$ & $0.91 * *$ & $10.53 * *$ & 0.12 \\
\hline
\end{tabular}

$*, * *=$ Significant at $5 \%$ and $1 \%$ probability levels, respectively.

\section{Variability, heritability and genetic advance}

An insight into the magnitude of variability exists in a crop species of most importance, as it provides the basis of the effective selection. In general, the genotypic coefficient of variability was higher than phenotypic coefficient of variability for all the thirteen characters under study which indicates that environment played a considerable role in the expression of their traits. The range of variability of different traits alone does not allow a decision as to which character was showing the highest degree of variability. Therefore, accurate relative comparison can be made with the help of phenotypic and genotypic co-efficient of variation. Phenotypic variation was partitioned into genotypic and environmental component.

The significant differences were observed among genotypes for all the characters studied. The higher magnitude of co-efficient of variation at genotypic as well as phenotypic levels observed for plant height, leaves per plant, leaf length, weight of clove and clove per bulb. Phenotypic variation was highest for plant height, leaf length, and cloves per bulb, weight of clove, leaf width and leaves per plant. Similar results were reported by Sharma et al. (1990), Lopez-Frasca et al.
(1997), Agrawal and Tiwari (2004) and Singh and Chand (2004).

Genotypic coefficient variation was also highest for clove per bulb followed leaf length. Korla et al. (1981), Mehta and Patel (1985) have also reported similar results in their studies. Moderate variation noted in case of weight of clove and diameter of bulb, while low GCV and PCV was observed for length of bulb. Moderate to low variation exerted for these traits revealed that there is a reasonable scope for improvement in these traits.

\section{Heritability and genetic advance}

Heritability in broad sense of a character is important to the breeder since it indicates the possibility and extent to which improvement is possible through selection. It also indicates direction of selection of selection pressure to be applied for the traits during selection because it measures relationship between parent and their progeny, widely used in determining the degree to which a character may be transmitted from parent to off spring. However, high heritability alone is not enough to make efficient selection in advanced generation unless accompanied by substantial amount of genetic advance (Burton and De-Vane, 1953). 
Table 2. Mean performance of sixty genotypes for thirteen characters in garlic.

\begin{tabular}{|c|c|c|c|c|c|c|c|c|c|c|c|c|c|}
\hline Genotypes & $\begin{array}{l}\text { Plant } \\
\text { height } \\
(\mathrm{cm})\end{array}$ & $\begin{array}{l}\text { Leaves/ } \\
\text { Plant }\end{array}$ & $\begin{array}{l}\text { Leaf } \\
\text { length } \\
(\mathrm{cm})\end{array}$ & $\begin{array}{l}\text { Leaf } \\
\text { width } \\
(\mathrm{cm})\end{array}$ & $\begin{array}{l}\text { Neck } \\
\text { thickness } \\
\text { of bulb } \\
\text { (cm) }\end{array}$ & $\begin{array}{l}\text { Diameter } \\
\text { of bulb } \\
(\mathbf{c m})\end{array}$ & $\begin{array}{l}\text { Bulb } \\
\text { yield/ } \\
\text { Plant (g) }\end{array}$ & $\begin{array}{l}\text { Cloves/ } \\
\text { Bulb }\end{array}$ & $\begin{array}{l}\text { Weight } \\
\text { of clove } \\
\text { (g) }\end{array}$ & $\begin{array}{l}\text { Length } \\
\text { of clove } \\
(\mathrm{cm})\end{array}$ & $\begin{array}{l}\text { Diameter } \\
\text { of clove } \\
(\mathbf{c m})\end{array}$ & $\begin{array}{l}\text { Length } \\
\text { of bulb } \\
\text { (cm) }\end{array}$ & $\begin{array}{l}\text { T.S.S } \\
(\%)\end{array}$ \\
\hline NDG-1 & 58.003 & 9.994 & 41.914 & 1.517 & 1.071 & 4.216 & 24.144 & 19.502 & 1.240 & 1.594 & 1.170 & 3.071 & 40.853 \\
\hline NDG-2 & 60.603 & 10.194 & 39.494 & 1.467 & 1.121 & 3.686 & 30.334 & 25.902 & 1.170 & 2.304 & 1.140 & 4.151 & 36.703 \\
\hline NDG-3 & 62.403 & 10.394 & 42.024 & 1.437 & 1.261 & 3.666 & 16.734 & 14.662 & 1.090 & 2.694 & 1.020 & 4.551 & 38.903 \\
\hline NDG-4 & 54.123 & 7.194 & 32.414 & 1.627 & 1.341 & 4.116 & 21.884 & 21.462 & 1.020 & 1.424 & 0.860 & 3.941 & 39.433 \\
\hline NDG-5 & 59.603 & 10.594 & 36.414 & 1.577 & 1.301 & 4.066 & 30.054 & 23.302 & 1.290 & 3.004 & 0.920 & 3.051 & 37.763 \\
\hline NDG-6 & 51.603 & 10.294 & 36.794 & 1.537 & 1.151 & 3.686 & 25.074 & 23.652 & 1.060 & 2.364 & 1.380 & 4.641 & 38.683 \\
\hline NDG-7 & 58.233 & 8.794 & 38.994 & 1.487 & 1.001 & 3.896 & 23.504 & 18.262 & 1.290 & 2.704 & 0.960 & 4.671 & 39.843 \\
\hline NDG-8 & 59.965 & 9.319 & 41.787 & 1.507 & 1.263 & 3.661 & 28.882 & 24.302 & 1.190 & 2.409 & 1.035 & 4.346 & 38.216 \\
\hline NDG-9 & 59.165 & 7.319 & 39.557 & 1.577 & 1.223 & 3.851 & 31.212 & 30.282 & 1.030 & 2.439 & 1.455 & 4.646 & 34.296 \\
\hline NDG-10 & 60.965 & 9.219 & 40.977 & 1.677 & 1.333 & 3.831 & 27.842 & 22.682 & 1.230 & 2.429 & 0.995 & 3.446 & 39.336 \\
\hline NDG-11 & 61.505 & 7.719 & 38.877 & 1.477 & 1.423 & 4.211 & 18.012 & 10.282 & 1.770 & 3.109 & 1.225 & 4.646 & 39.156 \\
\hline NDG-12 & 63.565 & 9.519 & 41.257 & 1.437 & 1.033 & 3.721 & 12.012 & 9.692 & 1.230 & 2.469 & 1.035 & 5.056 & 41.596 \\
\hline NDG-13 & 59.565 & 8.719 & 39.547 & 1.807 & 1.003 & 3.981 & 17.032 & 16.642 & 1.030 & 2.619 & 1.325 & 3.656 & 34.536 \\
\hline NDG-14 & 50.365 & 7.219 & 37.577 & 1.307 & 1.063 & 3.961 & 22.032 & 20.852 & 1.060 & 2.329 & 0.895 & 4.656 & 38.296 \\
\hline NDG-15 & 51.830 & 7.969 & 33.577 & 1.372 & 1.201 & 3.303 & 18.702 & 10.754 & 1.707 & 2.244 & 1.170 & 3.661 & 35.626 \\
\hline NDG-16 & 55.430 & 8.369 & 37.977 & 1.502 & 1.321 & 3.563 & 25.672 & 20.314 & 1.257 & 2.314 & 1.220 & 5.071 & 37.186 \\
\hline NDG-17 & 57.030 & 8.769 & 38.657 & 1.602 & 1.071 & 2.963 & 25.502 & 20.344 & 1.247 & 2.244 & 1.130 & 4.091 & 38.326 \\
\hline NDG-18 & 54.630 & 8.369 & 37.647 & 1.262 & 1.061 & 4.573 & 22.922 & 19.504 & 1.167 & 2.174 & 1.110 & 4.191 & 38.306 \\
\hline NDG-19 & 62.230 & 9.569 & 40.637 & 1.512 & 1.201 & 3.573 & 27.442 & 20.914 & 1.307 & 2.294 & 1.300 & 4.851 & 37.576 \\
\hline NDG-20 & 65.430 & 8.769 & 42.637 & 1.322 & 1.341 & 3.513 & 27.752 & 20.324 & 1.187 & 1.454 & 1.110 & 3.971 & 39.626 \\
\hline NDG-21 & 61.430 & 8.769 & 40.357 & 1.562 & 1.301 & 3.363 & 24.072 & 22.694 & 1.057 & 1.304 & 1.130 & 3.131 & 38.226 \\
\hline NDG-22 & 65.578 & 9.069 & 42.369 & 1.452 & 1.463 & 3.788 & 27.029 & 26.637 & 1.015 & 2.459 & 1.675 & 4.121 & 38.366 \\
\hline NDG-23 & 58.378 & 9.269 & 39.569 & 1.382 & 1.223 & 4.298 & 28.849 & 26.847 & 1.075 & 2.069 & 1.165 & 3.711 & 35.866 \\
\hline NDG-24 & 56.578 & 9.169 & 36.769 & 1.562 & 1.123 & 3.658 & 27.689 & 21.237 & 1.305 & 2.709 & 1.175 & 5.141 & 36.936 \\
\hline NDG-25 & 59.978 & 10.269 & 39.839 & 1.522 & 1.203 & 4.368 & 31.309 & 28.847 & 1.085 & 2.399 & 0.995 & 4.711 & 41.366 \\
\hline NDG-26 & 55.978 & 10.069 & 37.799 & 1.472 & 1.093 & 3.608 & 19.309 & 16.477 & 1.175 & 2.349 & 1.565 & 3.911 & 38.366 \\
\hline NDG-27 & 65.378 & 10.669 & 42.599 & 2.482 & 1.123 & 3.658 & 30.169 & 24.437 & 1.235 & 1.919 & 0.975 & 4.161 & 37.936 \\
\hline NDG-28 & 62.178 & 9.469 & 41.469 & 1.522 & 1.343 & 3.428 & 29.209 & 26.677 & 1.095 & 1.939 & 0.935 & 4.561 & 38.706 \\
\hline NDG-29 & 55.388 & 8.269 & 38.009 & 1.350 & 0.931 & 3.788 & 16.552 & 14.452 & 1.150 & 2.904 & 1.137 & 4.978 & 40.236 \\
\hline NDG-30 & 52.588 & 7.869 & 34.769 & 1.730 & 1.001 & 2.698 & 19.572 & 15.462 & 1.270 & 2.304 & 0.958 & 3.848 & 38.336 \\
\hline NDG-31 & 53.588 & 7.869 & 36.799 & 1.630 & 1.331 & 4.158 & 18.672 & 14.212 & 1.310 & 2.284 & 1.037 & 4.158 & 39.306 \\
\hline NDG-32 & 52.788 & 7.369 & 44.099 & 1.460 & 1.431 & 4.318 & 27.122 & 20.412 & 1.330 & 2.484 & 1.067 & 4.698 & 37.996 \\
\hline NDG-33 & 60.588 & 9.369 & 42.099 & 1.670 & 1.381 & 4.298 & 25.712 & 24.072 & 1.070 & 2.764 & 0.947 & 4.838 & 40.256 \\
\hline NDG-34 & 57.988 & 9.669 & 39.839 & 1.490 & 1.271 & 3.788 & 17.652 & 14.932 & 1.190 & 2.744 & 1.167 & 3.958 & 38.126 \\
\hline
\end{tabular}




\begin{tabular}{|c|c|c|c|c|c|c|c|c|c|c|c|c|c|}
\hline Genotypes & $\begin{array}{l}\text { Plant } \\
\text { height } \\
(\mathrm{cm})\end{array}$ & $\begin{array}{l}\text { Leaves/ } \\
\text { Plant }\end{array}$ & $\begin{array}{l}\text { Leaf } \\
\text { length } \\
(\mathrm{cm})\end{array}$ & $\begin{array}{l}\text { Leaf } \\
\text { width } \\
\text { (cm) }\end{array}$ & $\begin{array}{l}\text { Neck } \\
\text { thickness } \\
\text { of bulb } \\
\text { (cm) }\end{array}$ & $\begin{array}{l}\text { Diameter } \\
\text { of bulb } \\
\text { (cm) }\end{array}$ & $\begin{array}{l}\text { Bulb } \\
\text { yield/ } \\
\text { Plant (g) }\end{array}$ & $\begin{array}{l}\text { Cloves/ } \\
\text { Bulb }\end{array}$ & $\begin{array}{l}\text { Weight } \\
\text { of clove } \\
\text { (g) }\end{array}$ & $\begin{array}{l}\text { Length } \\
\text { of clove } \\
(\mathrm{cm})\end{array}$ & $\begin{array}{l}\text { Diameter } \\
\text { of clove } \\
(\mathbf{c m})\end{array}$ & $\begin{array}{l}\text { Length } \\
\text { of bulb } \\
\text { (cm) }\end{array}$ & $\begin{array}{l}\text { T.S.S } \\
(\%)\end{array}$ \\
\hline NDG-35 & 62.788 & 10.269 & 40.589 & 1.470 & 1.071 & 3.028 & 19.832 & 17.032 & 1.170 & 2.414 & 1.147 & 5.028 & 38.296 \\
\hline NDG-36 & 58.040 & 9.269 & 36.892 & 1.835 & 1.173 & 4.293 & 22.049 & 21.239 & 1.037 & 2.372 & 1.007 & 3.918 & 40.156 \\
\hline NDG-37 & 53.240 & 9.869 & 36.092 & 1.335 & 1.183 & 3.463 & 13.699 & 12.889 & 1.057 & 2.802 & 1.137 & 5.138 & 40.256 \\
\hline NDG-38 & 60.240 & 9.269 & 38.092 & 1.565 & 1.033 & 4.393 & 30.309 & 24.679 & 1.227 & 2.642 & 0.998 & 3.788 & 39.336 \\
\hline NDG-39 & 51.040 & 7.469 & 32.662 & 1.355 & 1.373 & 4.153 & 13.389 & 12.839 & 1.037 & 2.992 & 1.107 & 4.058 & 35.396 \\
\hline NDG-40 & 52.040 & 7.969 & 34.692 & 1.375 & 1.313 & 3.853 & 29.309 & 23.869 & 1.227 & 3.012 & 1.157 & 4.378 & 36.906 \\
\hline NDG-41 & 53.840 & 7.869 & 34.762 & 1.405 & 1.273 & 3.903 & 29.289 & 25.479 & 1.147 & 3.002 & 1.577 & 4.738 & 36.926 \\
\hline NDG-42 & 61.840 & 9.669 & 40.412 & 1.715 & 1.273 & 3.893 & 29.489 & 28.459 & 1.037 & 2.372 & 0.948 & 3.728 & 41.256 \\
\hline NDG-43 & 58.413 & 8.494 & 40.047 & 1.860 & 1.083 & 4.133 & 34.579 & 16.862 & 2.047 & 2.374 & 0.950 & 3.998 & 34.316 \\
\hline NDG-44 & 63.013 & 9.694 & 42.827 & 1.930 & 0.993 & 3.023 & 20.909 & 15.682 & 1.327 & 2.424 & 1.210 & 3.578 & 38.316 \\
\hline NDG-45 & 58.613 & 8.894 & 40.627 & 1.810 & 1.133 & 4.023 & 31.529 & 25.672 & 1.227 & 2.574 & 1.310 & 4.168 & 39.786 \\
\hline NDG-46 & 62.813 & 8.294 & 41.837 & 1.390 & 1.333 & 3.863 & 24.449 & 22.862 & 1.067 & 3.154 & 1.150 & 5.098 & 34.176 \\
\hline NDG--47 & 60.013 & 8.694 & 39.827 & 1.280 & 1.003 & 3.603 & 26.619 & 24.672 & 1.077 & 2.154 & 1.740 & 4.698 & 41.616 \\
\hline NDG-48 & 67.013 & 10.494 & 44.817 & 2.070 & 1.353 & 4.493 & 32.159 & 15.452 & 2.077 & 2.874 & 1.780 & 4.668 & 36.876 \\
\hline NDG-49 & 56.413 & 9.494 & 38.047 & 1.380 & 0.973 & 3.363 & 33.469 & 24.462 & 1.367 & 2.694 & 0.850 & 4.338 & 42.176 \\
\hline NDG-50 & 60.983 & 9.619 & 42.544 & 1.387 & 1.173 & 3.616 & 25.464 & 23.482 & 1.085 & 2.624 & 1.365 & 3.848 & 39.183 \\
\hline NDG-51 & 61.983 & 10.019 & 43.144 & 2.497 & 1.113 & 3.116 & 27.334 & 22.882 & 1.195 & 2.304 & 1.225 & 3.988 & 35.203 \\
\hline NDG-52 & 58.983 & 10.219 & 39.474 & 1.307 & 1.033 & 3.776 & 28.514 & 27.282 & 1.045 & 2.464 & 1.185 & 4.658 & 41.383 \\
\hline NDG-53 & 58.583 & 9.819 & 37.574 & 1.387 & 1.003 & 3.766 & 26.644 & 23.912 & 1.115 & 2.384 & 0.985 & 4.328 & 38.913 \\
\hline NDG-54 & 60.183 & 8.019 & 40.644 & 1.457 & 0.953 & 4.196 & 16.194 & 14.442 & 1.125 & 2.334 & 1.575 & 4.148 & 37.913 \\
\hline NDG-55 & 60.183 & 9.419 & 41.574 & 1.827 & 1.043 & 4.096 & 29.444 & 24.852 & 1.185 & 2.834 & 0.905 & 4.088 & 41.343 \\
\hline NDG56 & 58.383 & 8.419 & 39.894 & 1.497 & 1.053 & 4.116 & 29.694 & 17.442 & 1.705 & 2.624 & 0.805 & 3.768 & 39.143 \\
\hline G-41 & 58.565 & 8.438 & 38.818 & 1.821 & 1.121 & 3.050 & 30.955 & 23.036 & 1.344 & 2.624 & 1.303 & 3.967 & 38.731 \\
\hline G-50 & 57.816 & 7.463 & 37.416 & 1.356 & 1.071 & 3.348 & 31.097 & 20.457 & 1.520 & 2.335 & 1.232 & 3.827 & 38.530 \\
\hline G-282 & 62.194 & 9.800 & 41.316 & 1.549 & 1.194 & 3.837 & 28.374 & 24.429 & 1.161 & 2.711 & 1.076 & 4.265 & 40.382 \\
\hline $\begin{array}{l}\text { Punjab } \\
\text { Garlic }\end{array}$ & 59.916 & 8.575 & 38.897 & 1.422 & 1.117 & 3.629 & 33.230 & 30.945 & 1.074 & 2.456 & 1.649 & 4.162 & 40.799 \\
\hline Mean & 58.696 & 9.016 & 39.311 & 1.560 & 1.174 & 3.788 & 25.295 & 20.939 & 1.227 & 2.447 & 1.163 & 4.244 & 38.526 \\
\hline Std. Dev. & 3.917 & 0.962 & 2.735 & 0.246 & 0.139 & 0.407 & 5.577 & 5.098 & 0.225 & 0.385 & 0.228 & 0.520 & 1.948 \\
\hline Std. Error & 0.506 & 0.124 & 0.353 & 0.032 & 0.018 & 0.053 & 0.720 & 0.658 & 0.029 & 0.050 & 0.029 & 0.067 & 0.251 \\
\hline C.V. \% & 6.673 & 10.665 & 6.957 & 15.753 & 11.801 & 10.754 & 22.049 & 24.348 & 18.368 & 15.725 & 19.574 & 12.246 & 5.056 \\
\hline Lowest & 50.365 & 7.194 & 32.414 & 1.262 & 0.931 & 2.698 & 12.012 & 9.692 & 1.015 & 1.304 & 0.805 & 3.051 & 34.176 \\
\hline Highest & 67.013 & 10.669 & 44.817 & 2.497 & 1.463 & 4.573 & 34.579 & 30.945 & 2.077 & 3.154 & 1.780 & 5.141 & 42.176 \\
\hline
\end{tabular}




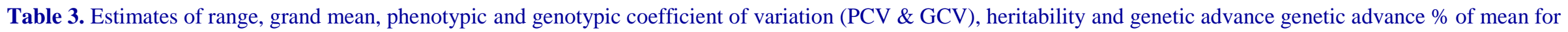
thirteen characters in garlic.

\begin{tabular}{|c|c|c|c|c|c|c|c|c|c|}
\hline \multirow[b]{2}{*}{ S. No. } & \multirow[b]{2}{*}{ Characters } & \multicolumn{2}{|l|}{ Range } & \multirow{2}{*}{$\begin{array}{l}\text { Grand } \\
\text { Mean }\end{array}$} & \multicolumn{2}{|c|}{ Coefficient of variation } & \multirow{2}{*}{$\begin{array}{l}\text { Heritability in } \\
\text { (broad sense) }\end{array}$} & \multirow{2}{*}{$\begin{array}{l}\text { Genetic } \\
\text { advance }\end{array}$} & \multirow{2}{*}{$\begin{array}{l}\text { Genetic } \\
\text { advance in } \\
\% \text { of mean }\end{array}$} \\
\hline & & Minimum & Maximum & & $\begin{array}{l}\text { Phenotypic } \\
\text { (PCV\%) }\end{array}$ & $\begin{array}{l}\text { Genotypic } \\
\text { (GCV \%) }\end{array}$ & & & \\
\hline 1 & Plant height $(\mathrm{cm})$ & 50.36 & 67.01 & 58.696 & 5.697 & 5.697 & 100.00 & 6.880 & 11.735 \\
\hline 2 & No of leaves per plant & 7.19 & 10.66 & 9.016 & 9.150 & 9.049 & 97.69 & 1.667 & 18.432 \\
\hline 3 & Length of leaf (cm) & 32.41 & 44.81 & 39.311 & 5.765 & 5.091 & 78.01 & 3.642 & 9.263 \\
\hline 4 & Width of leaf $(\mathrm{cm})$ & 1.26 & 2.49 & 1.560 & 13.405 & 13.379 & 99.62 & 0.429 & 27.508 \\
\hline 5 & Neck thickness of bulb (cm) & 0.93 & 1.46 & 1.174 & 10.141 & 9.457 & 86.97 & 0.214 & 18.169 \\
\hline 6 & Diameter of bulb (cm) & 2.69 & 4.57 & 3.788 & 8.884 & 8.702 & 95.94 & 0.669 & 17.558 \\
\hline 7 & Bulb Yield per plant (g) & 12.01 & 34.57 & 25.295 & 18.320 & 18.129 & 97.93 & 9.199 & 36.956 \\
\hline 8 & No of clove per bulb & 9.69 & 30.94 & 20.939 & 20.267 & 20.247 & 99.80 & 8.612 & 41.665 \\
\hline 9 & Weight of clove (g) & 1.01 & 2.07 & 1.227 & 15.402 & 15.284 & 98.47 & 0.382 & 31.243 \\
\hline 10 & Length of clove $(\mathrm{cm})$ & 1.30 & 3.15 & 2.447 & 13.137 & 12.621 & 92.29 & 0.609 & 24.977 \\
\hline 11 & Diameter of clove $(\mathrm{cm})$ & 0.80 & 1.78 & 1.163 & 18.021 & 12.689 & 49.58 & 0.212 & 18.404 \\
\hline 12 & Length of bulb (cm) & 3.05 & 5.14 & 4.244 & 10.400 & 10.368 & 99.39 & 0.906 & 21.292 \\
\hline 13 & Total soluble solids (\%) & 34.17 & 42.17 & 38.526 & 4.279 & 4.182 & 95.49 & 3.236 & 8.417 \\
\hline
\end{tabular}


The genetic advance is commonly predicted as a product of heritability ratio and selection differentials. Panse and Sukhatme (1967) mentioned that where high heritability value is accompanied by high genetic advance. The progress realized by selection would be most appropriate. In the present investigation, the highest estimates of heritability were observed in case of plant height and the highest genetic advance showed in bulb yield plant. Singh (1984) and Gupta et al. (2007) have also reported similar results in their studies. High heritability coupled with high genetic advance in per cent of mean was recorded for no of clove per bulb, bulb yield per plant, and weight of clove, width of leaf and length of clove. This indicates that these traits were less influenced by environment. Similar results were reported by Mehta and Patel (1985), Kumar et al. (2006) and Sonkiya et al. (2012).

\section{Conflict of interest statement}

Authors declare that they have no conflict of interest.

\section{Acknowledgement}

The work on garlic reported in this paper has been supported by research and teaching faculties of Department of Vegetable Science, N.D.U.A.T and We would also like to thank Mr. Murli Mohan Khetan for statistical analysis.

\section{References}

Agarwal, A., Tiwari, R.S., 2004. Genetic variability in garlic. Ind J. Agric. Sci. 74(3), 164-165.

Burton, G.W., de Vane, E.H., 1953. Estimated heritability in tall replicated clonal material. Agron. J. 45, 474-478.
Gupta, A.K., Samnotra, R.K., Kumar, S., 2007. Variability studies for some important horticultural traits in garlic (Allium sativum L.). Hary. J. Hort. Sci. 36(3/4), 301-302.

Janick, J., 1979. Horticultural Science. Freeman and Co., San Francisco. 544p.

Johnson, H.W., Robinson, H.F., Comstock, R.E., 1955. Estimates of genetic and environmental variability in soyabean. Agron. J. 47, 314-318.

Korla, B.N., Singh, A.K., Kalia, P., 1981. Genetic variability in garlic. Har. J. Horti. Sci. 10(1-2), 77-80.

Kothari, I.L., Shah, J.J., 1974. Histogenesis of seed stalk and inflorescence in garlic. Phytomorphol. 24, 42-48.

Kumar, A., Prasad, B., Saha, B.C., 2006. Genetic variability in garlic (Allium sativum L.). J. Interacademicia. 10(4), 467-472.

Lopez Frasca, A., Rigoni, C., Silvestri, V., Burba, J. L., Galmarini, C.R., 1997. Genetic variability estimation and correlation in white clonal type garlic characters. Acta Horticult. 433, 279-284.

Mehta, K.G., Patel, P.H., 1985. Genetic variability and path analysis in garlic. Madras Agric. J. 72(12), 691-695.

Panse, V.G., Shukhatme, P.V., 1967. Statistical Method for Agricultural Workers. $2^{\text {nd }}$ Edn. Indian Council of Agriculture Research, New Delhi (India).

Sharma, S. R., Kale, P. N., Dhankar, B.S., Shirsath, N.S., 1990. Variability and correlation studies in garlic. Har. J. Horti. Sci. 19(3-4), 313-317.

Singh, R.P., 1984. Association analysis in garlic. Madras Agric. J. 68 (9), 618-622.(1981).

Singh, Y., Ramesh, C., 2004. Genetic variability in garlic. Haryana J. Hortic. Sci. 33(1/2), 146-147.

Sonkiya, A.K., Singh, P.P., Naruka, I.S., 2012. Variability, character association and path coefficient analysis in garlic Medicinal Plants- Int. J. Phytomed. Related Indust. 4(2), 90-93.

\section{How to cite this article:}

Kumar, K., Ram, C.N., Yadav, G. C., Gautam, D. K., Kumar, P., Kumar, R., 2017. Studies on variability, heritability and genetic advance analysis for yield and yield attributes of garlic (Allium sativum L.). Int. J. Curr. Res. Biosci. Plant Biol. 4(4), 123-129. doi: https://doi.org/10.20546/ijcrbp.2017.404.019 